\title{
A Tight Lower Bound for Planar Multiway Cut with Fixed Number of Terminals
}

\author{
Dániel Marx* \\ Computer and Automation Research Institute, \\ Hungarian Academy of Sciences (MTA SZTAKI), \\ Budapest, Hungary \\ dmarx@cs.bme.hu
}

\begin{abstract}
Given a planar graph with $k$ terminal vertices, the PlanAR MultiwAY CUT problem asks for a minimum set of edges whose removal pairwise separates the terminals from each other. A classical algorithm of Dahlhaus et al. [2] solves the problem in time $n^{O(k)}$, which was very recently improved to $2^{O(k)} \cdot n^{O(\sqrt{k})}$ time by Klein and Marx [6]. Here we show the optimality of the latter algorithm: assuming the Exponential Time Hypothesis (ETH), there is no $f(k) \cdot n^{o(\sqrt{k})}$ time algorithm for Planar Multiway Cut. It also follows that the problem is W[1]-hard, answering an open question of Downey and Fellows [3].
\end{abstract}

\section{Introduction}

Multiway Cut (also called Multiterminal Cut) is a generalization of the classical minimum $s-t$ cut problem: given an undirected graph $G$ with subset $T$ of $k$ vertices specified as terminals, the task is to find a set of edges having minimum total weight whose deletion pairwise separates the $k$ terminal vertices from each other. While the problem is polynomial-time solvable for $k=2$, it becomes NP-hard for $k=3$ on general graphs. The special case of the problem on planar graphs, Planar Multiway CUt, is also NP-hard if $k$ can be arbitrarily large, but can be solved in time $O\left((4 k)^{k} n^{2 k-1} \log n\right)$ [2] or in time $O\left(k 4^{k} n^{2 k-4} \log n\right)$ [4]. That is, perhaps somewhat unexpectedly, the problem is polynomial-time solvable on planar graphs for every fixed $k$. In the companion paper [6], the dependence of the running time on the number of terminals was significantly improved: an algorithm with running time $2^{O(k)} \cdot n^{O(\sqrt{k})}$ was given for Planar Multiway Cut.

How much further the dependence on $k$ can be improved? Dahlhaus et al. [2] asked if Planar Multiway CUt can be solved in time $c^{k} \cdot n^{O(1)}$, which would be a significant improvement over all known algorithms. More generally, Downey and Fellows asked in the open problem list of their classical 1999 monograph [3] if the problem parameterized by the number of terminals is fixed-parameter

\footnotetext{
* Research supported by the European Research Council (ERC) grant "PARAMTIGHT: Parameterized complexity and the search for tight complexity results," reference 280152 .
} 
tractable, that is, can be solved in time $f(k) \cdot n^{O(1)}$ for some computable function $f$ depending only on $k$. The main result of the paper is a negative answer to this question: the problem is W[1]-hard parameterized by the number of terminals, making it unlikely to be fixed-parameter tractable. Moreover, our reduction shows that there is no $f(k) \cdot n^{o(\sqrt{k})}$ time algorithm for any computable function $f$, unless the Exponential Time Hypothesis (ETH) fails. ETH is the assumption that $n$-variable $m$-clause $3 \mathrm{SAT}$ cannot be solved in time $2^{o(n)} \cdot m^{O(1)}$, see $[5,7]$. Therefore, the $2^{O(k)} \cdot n^{O(\sqrt{k})}$ time algorithm of [6] is optimal in the sense that the exponent of $n$ cannot be better than $O(\sqrt{k})$. We present the hardness proof for the version of the problem where weights are allowed on the edges. However, the weights are polynomially large in the reductions, thus the results can be easily transferred to the case where each edge has unit weight by replacing an edge of weight $c$ by $c$ parallel edges (or parallel paths if one wishes to state the hardness result for simple graphs).

\section{The Reduction}

It will be convenient to present the reduction to Planar Multiway Cut from the following $\mathrm{W}[1]$-hard problem:

$$
\begin{aligned}
& \text { GRID TILING } \\
& \begin{aligned}
\text { Input: } & \text { Integers } k, n, \text { and } k^{2} \text { nonempty sets } S_{i, j} \subseteq[n] \times[n](1 \leq \\
& i, j \leq k) . \\
\text { Find: } & \text { For each } 1 \leq i, j \leq k, \text { a value } s_{i, j} \in S_{i, j} \text { such that } \\
& \quad-\text { If } s_{i, j}=(x, y) \text { and } s_{i, j+1}=\left(x^{\prime}, y^{\prime}\right), \text { then } x=x^{\prime} . \\
& \quad-\text { If } s_{i, j}=(x, y) \text { and } s_{i+1, j}=\left(x^{\prime}, y^{\prime}\right), \text { then } y=y^{\prime} .
\end{aligned}
\end{aligned}
$$

The W[1]-hardness of GRID TILING essentially follows from [8]. Note that the reduction transforms the problem of finding a $k$-clique into a $k \times k$ GRID TILING instance (we will need this fact for the tight lower bound in Corollary 5).

Lemma 1. GRID TILING is W[1]-hard parameterized by $k$.

To prove the W[1]-hardness of Planar Multiway Cut, we construct gadgets of the following form. An $n \times n$ gadget is an embedded planar graph $G_{n}$ with a set of $4 n+8$ distinguished vertices (see Figure 1). These distinguished vertices all appear on the boundary of the graph (i.e, on the infinite face) in the clockwise order $U L, u_{1}, \ldots, u_{n+1}, U R, r_{1}, \ldots, r_{n+1}, D R, d_{n+1}, \ldots, d_{1}, D L, \ell_{n+1}, \ldots, \ell_{1}$. Note that these distinguished vertices are a subset of the vertices on the boundary of the gadget, thus e.g., $u_{i}$ and $u_{i+1}$ are not necessarily adjacent. The four vertices $U L, U R, D R, D L$ are the only terminal vertices in the gadget. We say that a multiway cut $M$ of the gadget represents the pair $(x, y) \in[n]^{2}$ if $G_{n} \backslash M$ has four components that partition the distinguished vertices into the following classes:

$$
\begin{array}{ll}
\left\{U L, u_{1}, \ldots, u_{y}, \ell_{1}, \ldots, \ell_{x}\right\} & \left\{U R, u_{y+1}, \ldots, u_{n+1}, r_{1}, \ldots, r_{x}\right\} \\
\left\{D L, d_{1}, \ldots, d_{y}, \ell_{x+1}, \ldots, \ell_{n+1}\right\} & \left\{D R, d_{y+1}, \ldots, d_{n+1}, r_{x+1}, \ldots, r_{n+1}\right\}
\end{array}
$$

The main part of the hardness proof is to show that certain gadgets exist: 


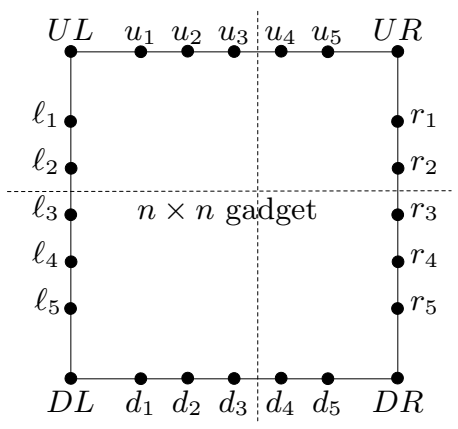

Fig. 1. The distinguished vertices of a $n \times n$ gadget for $n=4$. The dashed lines indicate a multiway cut of $U L, U R, D R, D L$ that represents the pair $(2,3)$.

Lemma 2. Given a subset $S \subseteq[n]^{2}$, we can construct in polynomial time a gadget $G_{S}$ and an integer $D$ such that the following properties hold:

1. For every $(x, y) \in S$, the gadget $G_{S}$ has a multiway cut of weight $D$ representing $(x, y)$.

2. If a multiway cut of $G_{S}$ has weight $D$, then it represents some $(x, y) \in S$.

3. Every multiway cut of $G_{S}$ has weight at least D.

The proof of Lemma 2 appears in Section 3. Assuming that such gadgets can be constructed, we can prove that Planar Multiway Cut is W[1]-hard.

Theorem 3. Planar Multiway Cut is W[1]-hard parameterized by the number of terminal vertices.

Proof. We reduce Grid Tiling to Planar Multiway Cut. Let $S_{i, j} \subseteq[n]^{2}$ $(1 \leq i, j \leq k)$ be the subsets in a GRID TILING instance. For every $1 \leq i, j \leq k$, we use Lemma 2 to construct the $n \times n$ gadget $G_{i, j}$ and compute the integer $D_{i, j}$ corresponding to the set $S_{i, j}$. Let $D=\sum_{1 \leq i, j \leq k} D_{i, j}$. We construct a planar graph $G$ by attaching the gadgets the following way:

- for every $1 \leq i \leq k, 1 \leq j<k$, we identify vertices $U R, r_{1}, \ldots, r_{n+1}, D R$ of $G_{i, j}$ with vertices of $U L, \ell_{1}, \ldots, \ell_{n+1}, D L$ of $G_{i, j+1}$, respectively, and

- for every $1 \leq i<k, 1 \leq j \leq k$, we identify vertices $D L, d_{1}, \ldots, d_{n+1}, D R$ of $G_{i, j}$ with vertices of $U L, u_{1}, \ldots, u_{n+1}, U R$ of $G_{i+1, j}$, respectively.

Note that we glue together the gadgets only at the distinguished vertices, not along the whole boundary. It is easy to see that $G$ is planar. With these identifications, the $4 k^{2}$ terminal vertices of the $k^{2}$ gadgets are identified into a set $T$ of exactly $(k+1)^{2}$ terminal vertices. For the sake of analysis, if there are two gadgets that have edges between two vertices $v$ and $u$, then we keep both edges as parallel edges in the graph $G$. This way, we can say the the edge set of $G$ is the disjoint union of the edge sets of all the gadgets. 


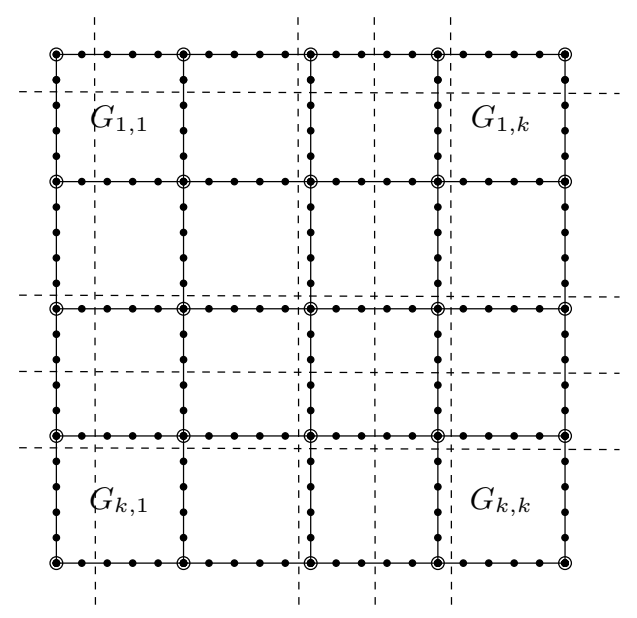

Fig. 2. Constructing the instance by identifying the distinguished vertices of $k^{2}$ gadgets. The circled vertices are terminals, the dashed lines indicate a multiway cut that corresponds to a clique.

We claim that there is a multiway cut of weight $D$ separating the terminals in $T$ if and only if the GRID TILING instance has a solution. Suppose first that $s_{i, j} \in S_{i, j}(1 \leq i, j \leq k)$ is a solution of the GRID TILING instance. By property 1 of Lemma 2 , every gadget $G_{i, j}$ has a multiway cut $M_{i, j}$ of weight $D_{i, j}$ that represents $s_{i, j}$. We claim that the union of these $M_{i, j}$ 's is a multiway cut separating $T$. This follows from the fact that the multiway cuts are consistent in the following sense: if distinguished vertices $v_{1}$ and $v_{2}$ of gadget $G_{i, j}$ are identified with vertices $v_{1}^{\prime}$ and $v_{2}^{\prime}$, respectively, of gadget $G_{i, j+1}$, then $v_{1}$ and $v_{2}$ are in the same component of $G_{i, j} \backslash M_{i, j}$ if and only if $v_{1}^{\prime}$ and $v_{2}^{\prime}$ are in the same component of $G_{i, j+1} \backslash M_{i, j+1}$. For example, consider vertices $r_{s_{1}}$ and $r_{s_{2}}$ of $G_{i, j}$, which are identified with vertices $\ell_{s_{1}}$ and $\ell_{s_{2}}$ of $G_{i, j+1}$. Suppose that $s_{i, j}=(x, y)$ and $s_{i, j+1}=\left(x^{\prime}, y^{\prime}\right)$ with $x=x^{\prime}$. Then from the fact that $M_{i, j}$ represents $(x, y)$, we have that $r_{s_{1}}$ and $r_{s_{2}}$ are in different components of $G_{i, j} \backslash M_{i, j}$ if and only if $s_{1} \leq x<s_{2}$. Similarly, $\ell_{s_{1}}$ and $\ell_{s_{2}}$ are in different components of $G_{i, j+1} \backslash M_{i, j+1}$ if and only if $s_{1} \leq x^{\prime}<s_{2}$. The consistency of the multiway cuts implies that if we look at a terminal vertex, say $D R$ of $G_{i, j}$, then its component in $G \backslash M$ is exactly the union of the component of $D R$ in $G_{i, j} \backslash M_{i, j}$, the component of $D L$ in $G_{i, j+1} \backslash M_{i, j+1}$, the component of $U R$ in $G_{i+1, j} \backslash M_{i, j}$, and the component of $U L$ in $G_{i+1, j+1} \backslash M_{i+1, j+1}$. Therefore, the terminals in $T$ are indeed separated from each other in $G \backslash M$.

For the other direction of the proof, suppose that $M$ is a multiway cut of $T$. In particular, this means that $M$ is a multiway cut of the four terminals of each gadget. Since the gadgets are edge disjoint, $M$ can be partitioned into disjoint sets $M_{i, j}(1 \leq i, j \leq k)$ such that $M_{i, j}$ is a multiway cut of gadget $G_{i, j}$. As every multiway cut of gadget $G_{i, j}$ has weight at least $D_{i, j}$ (Property 3 of 
Lemma 2) and $M$ has weight at most $D$, it follows that $M_{i, j}$ has weight exactly $D_{i, j}$. Therefore, by Property 2 of Lemma $2, M_{i, j}$ represents some set $s_{i, j} \in S_{i, j}$. We claim the the pairs $s_{i, j}$ form a solution for GRID TILING. We verify that if $s_{i, j}=(x, y)$ and $s_{i, j+1}=\left(x^{\prime}, y^{\prime}\right)$, then $x=x^{\prime}$. Suppose first that $x<x^{\prime}$. Then $r_{x+1}$ of $G_{i, j}$ is in the same component as $D R$ of $G_{i, j}$, while $\ell_{x+1}$ of $G_{i+1, j}$ (which is actually identified with $r_{x+1}$ of $G_{i, j}$ ) is in the same component as $U L$ of $G_{i, j+1}$ (as $x+1 \leq x^{\prime}$ ). Therefore, two terminal vertices are in the same component of $G \backslash M$, a contradiction. The case $x>x^{\prime}$, as well as the proof that $s_{i, j}$ and $s_{i+1, j}$ agree in the second component, is analogous.

To obtain a lower bound on the exponent of $n$ in the running time of algorithms for Planar Multiway Cut, we can use the following lower bound on Clique:

Theorem 4 ([1]). An $f(k) n^{o(k)}$ algorithm for CLIQUE implies that ETH fails.

Observe that, given an instance of CLIQUE, the two reductions in Lemma 1 and Theorem 3 create an instance of Planar Multiway Cut with $(k+1)^{2}$ terminals. Thus by Theorem 4, we have the following lower bound:

Corollary 5. If there is an $f(k) n^{o(\sqrt{k})}$ algorithm for PlanaR MUlTiWAY CUT, then ETH fails.

\section{Gadget construction}

The goal of this section is to construct a gadget that satisfies the requirements of Lemma 2. Section 3.1 describes the construction of the gadget, Section 3.2 proves Property 1 of Lemma 2 by showing how a pair $(x, y) \in S$ defines a cheap multiway cut, while Section 3.3 proves Properties 2 and 3 by showing how a cheap multiway cut defines a pair in $S$.

\subsection{Construction}

Let $N:=n^{2}+2 n+1$. We start the construction of the gadget $G_{S}$ with an $(N+1) \times(N+1)$ grid: let us introduce vertices $g[i, j](0 \leq i, j \leq N)$ such that vertices $g[i, j]$ and $g\left[i^{\prime}, j^{\prime}\right]$ are adjacent if and only if $\left|i-i^{\prime}\right|+\left|j-j^{\prime}\right|=1$. The grid is pictured as $g[0,0]$ being the upper left corner, $g[0, N]$ the upper right corner, etc. We call the edges on the horizontal path from $g[i, 0]$ to $g[i, N]$ the row $R_{i}$, while the edges on the vertical path from $g[0, j]$ to $g[N, j]$ is the column $C_{j}$. We also say that the horizontal edge $\{g[i, j], g[i, j+1]\}$ of row $R_{i}$ has column number $j$ and the vertical edge $\{g[i, j], g[i+1, j]\}$ of column $C_{j}$ has row number $i$.

For ease of notation, we define the functions $\alpha(s)=N-n-2+s$ and $\beta(x, y)=x+y n$ (observe that $\beta(n+1, y)=\beta(1, y+1)$ and $n+1 \leq \beta(x, y) \leq$ $n^{2}+n=N-n-1=\alpha(1)$ for every $\left.1 \leq x, y \leq n\right)$. The distinguished vertices of the gadget are defined as follows (see Figure 3 ): for every $1 \leq s \leq n+1$, we set

$$
\begin{aligned}
& U L=g[0,0] \quad U R=g[0, N] \quad u_{s}=g[0, \beta(1, s)] d_{s}=g[N, \beta(1, s)] \\
& D L=g[N, 0] D R=g[N, N] \ell_{s}=g[\alpha(s), 0] \quad r_{s}=g[s, N] .
\end{aligned}
$$




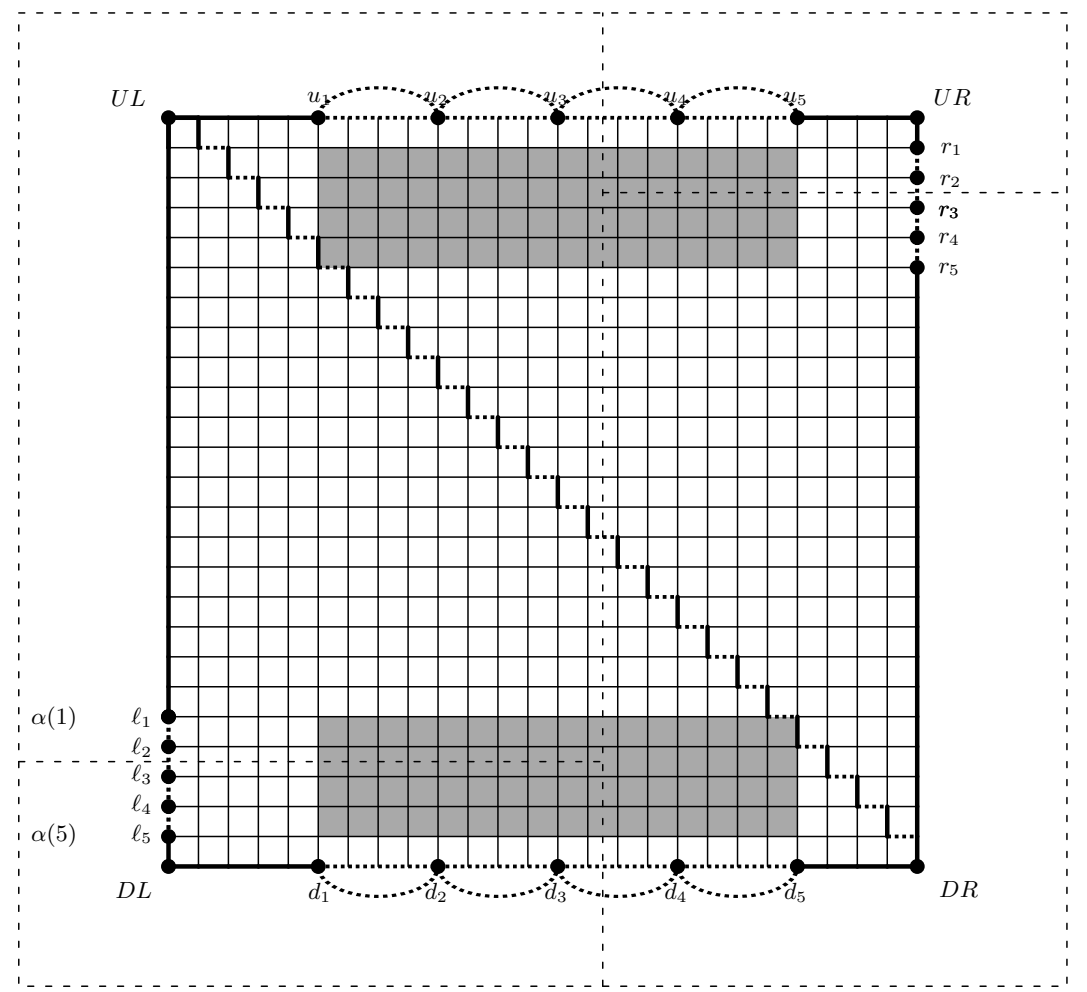

Fig. 3. A $n \times n$ gadget with $n=4$ (and hence $N=n^{2}+2 n+1=25$ ). The black dots represent the distinguished vertices, strong edges have weight $\infty$, dotted strong edges have weight at least $W^{3}$, normal edges have weight between $W^{2}$ and $W^{2}+N W$. Some of the shaded cells are marked special (the cell edges are omitted from the figure). The dashed lines show the 4 components created by the multiway cut corresponding to the pair $(2,3)$.

We refer to the edges of the grid as grid edges. We define now the weight of the grid edges. Let $W:=100 N^{2}$. Let us set the weights first as follows:

- For $0 \leq i \leq N, 0 \leq j \leq N-1$, vertical edge $\{g[i, j], g[i+1, j]\}$ has weight

$$
\begin{cases}\infty & \text { if } i=j-1, \\ \infty & \text { if } i=0 \text { and } j \notin[\alpha(1), \alpha(n)], \\ W^{3}+W^{2} & \text { if } i=0 \text { and } j \in[\alpha(1), \alpha(n)], \\ \infty & \text { if } i=N \text { and } j \notin[1, n], \\ W^{3}+W^{2} & \text { if } i=N \text { and } j \in[1, n], \\ W^{2} & \text { otherwise. }\end{cases}
$$


- For $0 \leq i \leq N-1,0 \leq j<N-1$, horizontal edge $\{g[i, j], g[i, j+1]\}$ has weight

$$
\begin{cases}\infty & \text { if } i=0 \text { and } j \notin[\beta(1,1), \beta(n, n)], \\ W^{3}+W^{2}+j W & \text { if } i=0 \text { and } j \in[\beta(1,1), \beta(n, n)], \\ W^{2}+j W & \text { if } 0<i<j, \\ W^{3}+W^{2}-i^{2} W-(N-i)^{2} W & \text { if } i=j, \\ W^{2}+(N-j) W & \text { if } j<i<N, \\ W^{3}+W^{2}+(N-j) W & \text { if } j=N \text { and } j \in[\beta(1,1), \beta(n, n)], \\ \infty & \text { if } j=N \text { and } j \notin[\beta(1,1), \beta(n, n)] .\end{cases}
$$

Note that the only part of the boundary with finite edges are the horizontal path between $u_{1}$ and $u_{n+1}$, the horizontal path between $d_{1}$ and $d_{n+1}$, the vertical path between $\ell_{1}$ and $\ell_{n+1}$, and the vertical path between $r_{1}$ and $r_{n+1}$.

Let us consider a column number $\beta(1,1) \leq z \leq \beta(n, n)$. The horizontal edges with column number $z$ have weight either $W^{2}$ or $W^{3}+W^{2}$ plus or minus some lower-order terms. If we sum the weights of these horizontal edges, then the extra terms $j W$ in rows less than $z$ and the extra terms $(N-j) W$ for rows greater than $z$ are canceled by the negative terms in the weight of the edge in row $R_{z}$. Thus the total weight of these edges is the same for every column number $z$.

Claim 6 For every $\beta(1,1) \leq z \leq \beta(n, n)$, the total weight of all the horizontal edges with column number $z$ is exactly $3 W^{3}+(N+1) \cdot W^{2}$.

For every $1 \leq s \leq n$, we add the upper ear edge $\left\{u_{s}, u_{s+1}\right\}$ and the lower ear edge $\left\{d_{s}, d_{s+1}\right\}$, both having weight $W^{3}$.

The cell $C[i, j]$ is the face of the grid with the corners $g[i, j], g[i+1, j], g[i+$ $1, j+1], g[i, j+1]$ on its boundary. We mark each cell either as normal or special and add edges to the cell accordingly (we will call these new edges the cell edges). If the cell $C[i, j]$ is normal, then we add new (parallel) edges $\{g[i, j], g[i+1, j]\}$, $\{g[i+1, j], g[i+1, j+1]\},\{g[i+1, j+1], g[i, j+1]\},\{g[i, j+1], g[i, j]\}$, all with weight 2 (see Figure 4 ). If the cell $C[i, j]$ is special, then we add the edges $\{g[i, j], g[i+1, j]\}$ and $\{g[i, j+1], g[i+1, j+1]\}$ having weight 1 , as well as the edges $\{g[i, j], g[i, j+1]\},\{g[i+1, j], g[i, j+1]\}$ having weight 2 .

The crucial properties of the cell edges are the following. If the two upper corners $g[i, j], g[i, j+1]$ are separated from the two lower corners $g[i+1, j]$, $g[i+1, j+1]$, then the cell edges connecting these vertices have to be in the multiway cut. Observe that the total weight of these cell edges is exactly 4 both in a normal cell and in a special cell. Similarly, if the two corners $g[i, j], g[i+1, j]$ on the left are separated from the two corners $g[i, j+1], g[i+1, j+1]$ on the right, then the weight of the edges that need to be in the multiway cut is exactly 4 for both type of cells. However, there is a difference if we want to partition the four corners of the cell $C[i, j]$ into three components $\{g[i, j]\},\{g[i+1, j]\}$, $\{g[i, j+1], g[i+1, j+1]\}$. For normal cells, the edges $\{g[i, j], g[i+1, j]\},\{g[i+$ $1, j], g[i+1, j+1]\},\{g[i, j+1], g[i, j]\}$, having total weight 6 , need to be in the multiway cut. On the other hand, for special cells, the edges $\{g[i, j], g[i+1, j]\}$, 

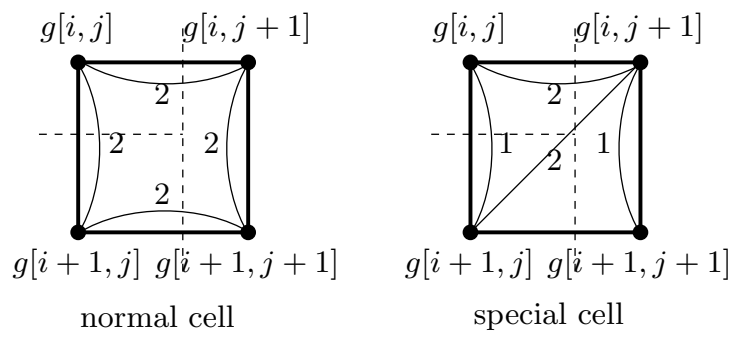

Fig. 4. The cell edges within a normal and a special cell. The strong edges are the 4 grid edges forming the boundary of the cell. The dashed lines show a 3-way partition of the corners that costs 6 in a normal cell and only 5 in a special cell.

$\{g[i, j], g[i, j+1]\},\{g[i+1, j], g[i, j+1]\}$ that need to be in the multiway cut have total weight 5 . Similarly, a multiway cut with partition $\{g[i, j], g[i+1, j]\}$, $\{g[i, j+1]\},\{g[i+1, j+1]\}$ need to contain cell edges of total weight 6 in a normal cell, but weight 5 is sufficient in a special cell.

We complete the construction of the gadget by encoding the set $S$ : for every $(x, y) \in S$, we mark the two cells $C[x, \beta(x, y)]$ and $C[\alpha(x), \beta(x, y)]$ special. Finally, we set $D:=7 W^{3}+(2 N+2) W^{2}+4(2 N-3)+10<8 W^{3}$.

\subsection{Pair $(x, y) \Rightarrow$ multiway cut.}

Given a pair $(x, y) \in S$, we construct a multiway cut $M$ representing $(x, y)$ the following way. We partition the vertices of gadget the following way: vertex $g[i, j]$ is in the same class as

- UL if $i \leq \alpha(x)$ and $j \leq \beta(x, y)$,

- UR if $i \leq x$ and $j>\beta(x, y)$,

- DL if $i>\alpha(x)$ and $j \leq \beta(x, y)$,

- DR if $i>x$ and $j>\beta(x, y)$.

Observe that this partition indeed represents the pair $(x, y)$. The multiway cut $M$ contains all the edges that connect edges between two different classes:

1. ear edges $\left\{u_{y}, u_{y+1}\right\},\left\{d_{y} d_{y+1}\right\}$,

2. $\{g[s, \beta(x, y)], g[s, \beta(x, y)+1]\}$ for every $0 \leq s \leq N$,

3. $\{g[\alpha(x), s], g[\alpha(x)+1, s]\}$ for every $0 \leq s \leq \beta(x, y)$,

4. $\{g[x, s], g[x+1, s]\}$ for every $\beta(x, y)<s \leq N$,

5 . and some number of cell edges.

The total weight of the two edges in the first group is $2 W^{3}$. The second group contains all the horizontal edges with column number $\beta(x, y)$, hence their total weight is exactly $3 W^{3}+(N+1) \cdot W^{2}$ by Claim 6 . Groups 3 and 4 contain $N+1$ vertical edges in total, two of them having weight $W^{3}+W^{2}$ and the rest having weight $W^{2}$. Note that none of these edges has weight $\infty$ : it is not possible that $\alpha(x)=s-1$ for some $s \leq \beta(x, y)($ as $\alpha(x) \geq N-n-1$ and $\beta(x, y) \leq N-n-1)$ 
or that $x=s-1$ for some $s>\beta(x, y)$ (as $x \leq n$ and $\beta(x, y) \geq n+1)$. Thus the total weight of the edges in Groups 3 and 4 is $2 W^{3}+(N+1) W^{2}$. We can conclude that the total weight of the grid edges and ear edges in the multiway cut is $7 W^{3}+(2 N+2) W^{2}$.

What remains to be shown is that the weight of the cell edges in $M$ is at most $4(2 N-3)+10$ in the multiway cut. Let us analyze how the corners of the cell $C[i, j]$ are partitioned by the multiway cut.

1. Horizontal cut: $\{g[i, j], g[i, j+1]\},\{g[i+1, j], g[i+1, j+1]\}$ if $i=\alpha(x)$ and $j<\beta(x, y)$ holds, or if $i=x$ and $j>\beta(x, y)$ holds.

2. Vertical cut: $\{g[i, j], g[i+1, j]\},\{g[i, j+1], g[i+1, j+1]\}$ if $j=\beta(x, y)$ and $i \notin\{x, \alpha(x)\}$.

3. 3-way $\dashv$ cut: $\{g[i, j]\},\{g[i+1, j]\},\{g[i, j+1], g[i+1, j+1]\}$ if $i=\alpha(x)$ and $j=\beta(x, y)$.

4. 3-way $\vdash$ cut: $\{g[i, j], g[i+1, j]\},\{g[i, j+1]\},\{g[i+1, j+1]\}$ if $i=x$ and $j=\beta(x, y)$.

5. All corners are in the same class otherwise.

For each cell in the first two groups, the weight of the cell edges in the multiway cut is exactly 4 (regardless if the cell is normal or special). As $(x, y) \in S$, the cells $C[\alpha(x), \beta(x, y)], C[x, \beta(x, y)]$ were marked as special. Therefore, each of the two cells in groups 3 and 4 contribute weight 5 to the multiway cut. It follows that the total weight of the cell edges in $M$ is exactly $4(2 N-3)+10$, as required. This proves Property 1 of Lemma 2.

\subsection{Multiway cut $\Rightarrow$ pair $(x, y)$.}

To prove Properties 2 and 3 of Lemma 2, consider a multiway cut $M$ of weight at most $D$. We prove that $M$ has to be of the form shown in Figure 3: it consists of a "vertical cut" with two "horizontal cuts" on its two sides. Moreover, the the two cells where these cuts meet should be special. Taking into account the way in which the special cells are located, we can conclude that the two horizontal cuts have the same "vertical position" and that the pair $(x, y)$ is indeed in $S$.

Let us denote by $K_{U L}$ the component of $G \backslash M$ containing the vertex $U L$ (and we define $K_{U R}$ etc. similarly). We call the path $U L=g[0,0], g[0,1], g[1,1]$, $g[1,2], \ldots, g[N-1, N-1], g[N-1, N], D R=g[N, N]$ the diagonal path.

Claim 7 Multiway cut $M$ contains exactly one upper ear edge, exactly one lower ear edge, exactly one edge of the diagonal path, and exactly edge from each of $C_{0}, C_{N}, R_{0}$, and $R_{N}$.

Proof. The vertex $u_{1}$ is in $K_{U L}$ (as horizontal edges of weight $\infty$ connect it to $U L)$ and $u_{n+1}$ is in $K_{U R}$. This means that at least one of the upper ear edges is in $M$. Similarly, at least one of the lower ear edges have to be in $M$. It is also clear that $M$ has to contain at least one edge from the diagonal path and each of $C_{0}$, $C_{N}, R_{0}$, and $R_{N}$, as each one of these 5 edge sets connects two distinct terminals. Every edge shared by these 5 sets has weight $\infty$ (note that edge $\{g[0,0], g[0,1]\}$ 
appears both on the diagonal path and $R_{0}$, while $\{g[N-1, N], g[N, N]\}$ appears both on the diagonal path and $C_{N}$ ). Therefore, $M$ contains at least 5 edges from these 5 sets. As every ear edge and every edge in these 5 sets have weight either $\infty$ or at least $W^{3}$ and the weight of $M$ is at most $D<8 W^{3}$, the multiway cut contains exactly one edge from each of these sets.

By Claim 7, the multiway cut $M$ contains

$-\left\{u_{y_{1}}, u_{y_{1}+1}\right\}$ for some $1 \leq y_{1} \leq n$,

$-\left\{d_{y_{2}}, d_{y_{2}+1}\right\}$ for some $1 \leq y_{2} \leq n$,

$-\left\{\ell_{x_{1}}, \ell_{x_{1}+1}\right\}$ for some $1 \leq x_{1} \leq n$,

$-\left\{r_{x_{2}}, r_{x_{2}+1}\right\}$ for some $1 \leq x_{2} \leq n$,

$-\left\{g\left[0, z_{1}\right], g\left[0, z_{1}+1\right]\right\}$ for some $\beta(1,1) \leq z_{1} \leq \beta(n, n)$,

$-\left\{g\left[N, z_{2}\right], g\left[N, z_{2}+1\right]\right\}$ for some $\beta(1,1) \leq z_{2} \leq \beta(n, n)$, and

$-\{g[z, z], g[z, z+1]\}$ for some $0<z<N$.

As $C_{0}$ contains exactly one edge of the multiway cut, every vertex on $C_{0}$ is in $K_{U L} \cup K_{D L}$. We can argue similarly for the other three sides.

Claim $8 V\left(C_{0}\right) \subseteq K_{U L} \cup K_{D L}, V\left(C_{N}\right) \subseteq K_{U R} \cup K_{D R}, V\left(R_{0}\right) \subseteq K_{U L} \cup K_{U R}$, and $V\left(R_{N}\right) \subseteq K_{D L} \cup K_{D R}$.

Observe that every horizontal grid edge on the diagonal path has weight at least $W^{3}+W^{2} / 2$. Therefore, the 7 edges given by Claim 7 (4 on the boundary of the grid, 1 on the diagonal path, and 2 ear edges) have total weight at least $7 W^{3}+4 W^{2}+W^{2} / 2$. This implies that the remaining edges have total weight less than $(2 N-2) W^{2}$, otherwise the weight would be at least $7 W^{3}+(2 N+$ 2) $W^{2}+W^{2} / 2>D$. In particular, $M$ can contain at most $2 N-3$ further grid edges, that is, the total number of grid edges in $M$ is at most $2 N+2$.

Claim $9 M$ contains exactly one edge from each of $R_{i}$ and $C_{i}(0 \leq i, j \leq N)$.

Proof. As $V\left(C_{0}\right) \subseteq K_{U L} \cup K_{D L}$ and $V\left(C_{N}\right) \subseteq K_{U R} \cup K_{D R}$ (by Claim 8), the multiway cut $M$ has to contain at least one edge of $R_{i}$ for every $1 \leq i \leq N-1$. In a similar way, $M$ contains at least one edge $C_{j}$ for every $1 \leq j \leq N-1$. As $M$ contains at most $2 N+2$ grid edges, it immediately follows that $M$ contains exactly one edge of each row and column.

Observe that every vertical grid edge inside the grid has weight exactly $W^{2}$ if its weight is finite. Therefore, we know the exact weight of the vertical grid edges in $M$ and hence can bound the total weight of the horizontal grid edges.

Claim 10 The total weight of vertical grid edges in $M$ is exactly $2 W^{3}+(N+$ 1) $W^{2}$ and therefore the total weight of horizontal grid edges in $M$ is at most $3 W^{3}+(N+1) W^{2}+4(2 N-3)+10<3 W^{3}+(N+1) W^{2}+W$.

Claim $11 M$ contains $\{g[i, z], g[i, z+1]\}$ for every $0 \leq i \leq N$ and the total weight of the horizontal grid edges in $M$ is exactly $3 W^{3}+(N+1) W^{2}$. 
Proof. Since $\{g[z, z], g[z, z+1]\}$ is the unique edge of the multiway cut that is on the diagonal path from $U L$ to $D R$, we have $g[z, z] \in K_{U L}$ and $g[z, z+1] \in K_{D R}$. Therefore, the multiway cut has to contain an edge of the vertical path from $g[z, z] \in K_{U L}$ to $g[N, z] \in R_{N} \subseteq K_{D L} \cup K_{D R}$ (by Claim 8). Since we know that the multiway cut $M$ contains exactly one edge of $C_{z}$ (Claim 9), it follows that $M$ does not contain any edge of the vertical path from $g[z, z]$ to $g[0, z]$, that is, every vertex on this path is in $K_{U L}$. The multiway cut has to separate the vertices on this vertical path from the vertices of $C_{N} \subseteq K_{U R} \cup K_{D R}$ (Claim 8), thus for every $0 \leq i<z$ the unique edge in $M \cap R_{i}$ has column number at least $z$. Therefore, the total weight of these $z$ edges is at least $W^{3}+z W^{2}+z^{2} W$, with equality only if every edge has column number exactly $z$ (and $\beta(1,1) \leq z \leq \beta(n, n)$ to ensure that $\{g[0, z], g[0, z+1]\}$ has finite weight). A similar argument shows that every edge in $M \cap R_{i}$ for $i>z$ has to have column number at most $z$, and hence the total weight of these $N-z$ edges is at least $W^{3}+(N-z) W^{2}+(N-z)^{2} W$ with equality only if all these edges have the same column number $\beta(1,1) \leq z \leq \beta(n, n)$. Taking into account also the edge $\{g[z, z], g[z, z+1]\}$, we get that the total weight of the horizontal grid edges is at least $3 W^{3}+(N+1) \cdot W^{2}$, with equality only if all of them have column number $z$. Furthermore, as the weight of every grid edge is a multiple of $W$, if not every horizontal edge has column number $z$, then the weight is at least $3 W^{2}+(N+1) W^{2}+W$, contradicting Claim 10. Thus every horizontal edge has the same column number $\beta(1,1) \leq z \leq \beta(n, n)$.

For $i=0$ and $i=N$, Claim 11 implies $z=z_{1}=z_{2}$ and hence $\beta(1,1) \leq z \leq$ $\beta(n, n)$ (to avoid the selection of edges with weight $\infty$ on the boundary).

Claim 12 The unique edge of $M \cap C_{j}$ is $\left\{g\left[x_{1}, j\right], g\left[x_{1}+1, j\right]\right\}$ if $j \leq z$ and $\left\{g\left[x_{2}, j\right], g\left[x_{2}+1, j\right]\right\}$ if $j>z$.

Proof. Observe that for every $i \leq x_{1}$ and $j \leq z$, vertex $g[i, j]$ is in $K_{U L}$ : the multiway cut does not contain any of the edges on the vertical path from $U L$ to $g[i, 0]$ and on the horizontal path from $g[i, 0]$ to $g[i, j]$. Similarly, vertex $g[i, j]$ is in $K_{D L}$ if $i>x_{1}$ and $j \leq z$. These two statements together imply that the edge $\left\{g\left[x_{1}, j\right], g\left[x_{1}+1, j\right]\right\}$ has to be in the multiway cut for every $0 \leq j \leq z$. The argument for $j>z$ is analogous.

By Claim 12, the $2 N+2$ grid edges in the multiway cut are arranged as in Figure 3. It follows that the multiway cut contains cell edges from exactly $2 N-1$ cells. As the weight of $M$ is at most $D$, the total weight of these cell edges is at most $4(2 N-3)+10$.

Claim 13 The cells edges in $M$ have total weight exactly $4(2 N-3)+10$ and the cells $C\left[x_{1}, z\right]$ and $C\left[x_{2}, z\right]$ are special.

Proof. For every $0 \leq j<z$, the two upper corners of the cell $C\left[x_{1}, j\right]$ are separated from the two lower corners. Therefore, the weight of the cell edges from $C\left[x_{1}, j\right]$ in the multiway cut is 4 (no matter whether the cell is special or not). The same is true for every cell $C\left[x_{2}, j\right]$ with $j>z$. For the cells $C[i, z]$ 
with $i \notin\left\{x_{1}, x_{2}\right\}$, the two corners on the left are separated from the two corners on the right, which again means that the weight contributed by the cell edges of $C[i, z]$ is 4 . Thus the weight contributed by the cell edges of these $2 N-3$ cells is at least $4(2 N-3)$. As the total weight of the cell edges is at most $4(2 N-3)+10$, the contribution of the two cells $C\left[x_{1}, z\right]$ and $C\left[x_{2}, z\right]$ is at most 10. The corners of $C\left[x_{1}, z\right]$ are partitioned as $\left\{g\left[x_{1}, z\right]\right\},\left\{g\left[x_{1}+1, z\right]\right\}$, $\left\{g\left[x_{1}, z+1\right], g\left[x_{1}+1, z+1\right]\right\}$ by the multiway cut, while the corners of $C\left[x_{2}, z\right]$ are partitioned as $\left\{g\left[x_{2}, z\right], g\left[x_{2}+1, z\right]\right\},\left\{g\left[x_{2}, z+1\right]\right\},\left\{g\left[x_{1}+1, z+1\right]\right\}$. In both cases, the weight of the edges contained in the multiway cut is 5 if the cell is special and 6 if it is normal. Therefore, both of these cells have to be special.

Suppose that $z=\beta(x, y)$ for some $1 \leq x, y \leq n$ (recall that $\beta(1,1) \leq z \leq$ $\beta(n, n))$. There are at most two cells with column number $z$ that are special. By construction, $C\left[x_{1}, z\right]$ and $C\left[x_{2}, z\right]$ are special only if $(x, y) \in S$ and we have $x_{1}=$ $\alpha(x), x_{2}=x$. That is, the multiway cut $M$ contains the edge $\{g[\alpha(x), 0], g[\alpha(x)+$ $1,0]\}=\left\{\ell_{x}, \ell_{x}+1\right\}$ and the edge $\{g[x, N], g[x+1, N]\}=\left\{r_{x}, r_{x}+1\right\}$. Finally, as the multiway cut contains $\{g[0, z], g[0, z+1]\}$ and $\{g[N, z], g[N, z+1]\}$, the two ear edges contained in the multiway cut should be $\left\{u_{y}, u_{y}+1\right\}$ and $\left\{d_{y}, d_{y}+1\right\}$. This proves that the multiway cut represents the pair $(x, y) \in S$, what we had to show. Claim 13 also shows that there is no multiway cut with weight $<D$.

\section{References}

1. J. Chen, X. Huang, I. A. Kanj, and G. Xia. Strong computational lower bounds via parameterized complexity. Journal of Computer and System Sciences, 72(8):13461367, 2006.

2. E. Dahlhaus, D. S. Johnson, C. H. Papadimitriou, P. D. Seymour, and M. Yannakakis. The complexity of multiterminal cuts. SIAM J. Comput., 23(4):864-894, 1994.

3. R. G. Downey and M. R. Fellows. Parameterized Complexity. Monographs in Computer Science. Springer, New York, 1999.

4. D. Hartvigsen. The planar multiterminal cut problem. Discrete Applied Mathematics, 85(3):203-222, 1998.

5. R. Impagliazzo, R. Paturi, and F. Zane. Which problems have strongly exponential complexity? J. Comput. System Sci., 63(4):512-530, 2001.

6. P. N. Klein and D. Marx. Solving planar $k$-terminal cut in time $O\left(n^{c \sqrt{k}}\right)$. To appear in ICALP 2012.

7. D. Lokshtanov, D. Marx, and S. Saurabh. Lower bounds based on the Exponential Time Hypothesis. Bulletin of the EATCS, 84:41-71, 2011.

8. D. Marx. On the optimality of planar and geometric approximation schemes. In FOCS 2007, pages 338-348, 2007. 